THE GROWTH OF VARIOUS SPECIES OF BACTERIA AND OTHER MICRO-ORGANISMS IN ATMOSPHERES ENRICHED WITH OXYGEN ${ }^{1}$

By BENJAMIN MOORE, M.A., D.Sc., Johnston Professor of BioChemistry, and R. STENHOUSE WILLIAMS, M.B., D.P.H., Assistant Lecturer on Public Health Bacteriology, University of Liverpool.

From the Bio-Chemical and Public Health Laboratories, University of Liverpool

(Received May 19th, 1910)

In a previous paper, ${ }^{2}$ we have described experiments showing that the Bacillus tuberculosis will only grow between certain percentages of oxygen in the atmosphere to which it is exposed, and is killed either by absence of oxygen or by over eighty per cent. of oxygen. In other words, a partial pressure of oxygen of about $600 \mathrm{~mm}$. of mercury is fatal to Bacillus tuberculosis equally with a very low pressure.

It was also found that such a high oxygen pressure stopped the growth of the organism Staphylococcus pyogenes aureus, and usually considerably inhibited, or stopped in some cases, that of $S$. albus; the third organism of this group, S. citreus, was less obviously affected.

Only twice have we obtained a fair growth of $S$. aureus in increased oxygen; as a rule, the contrast between the control tubes grown in air and the oxygen-grown tubes is most striking, the oxygen-grown tubes appearing to be quite sterile, while the others show a luxuriant growth.

There was this important difference, however, that the B. tuberculosis was killed outright by the oxygen, the small patches of started growths having turned dark brown at the end of the more prolonged period of about two to three weeks necessary to test the culture of this more slowly growing organism, while the controls were white and flourishing. On the other hand, at the end of the shorter period of one to two days necessary to test the culture of the Staphylococci, although the oxygen tubes had not grown, if they were now transferred to ordinary air a fairly good growth was obtained.

In the former paper a fair number of other organisms were similarly tested, such as B. coli, B. typhosus, B. pyocyaneus, B. dysenteriae

1. Towards the expenses of this research a Grant was made by the British Medical Association.

2. This Journal, Vol. IV, p. 177, 1909. An important erratum occurs in this paper. At p. 183 , line 21 , transpose the words 'oxygen' and ' air.' 
(Flexner), B. dysenteriae (Shiga), B. dysenteriae (Kruse), B. diphtheriae, $V$. cholerae; and it was found that these were practically not affected by the higher oxygen percentages.

Since this aspect of the subject, namely, the effects of increased oxygen has not been nearly as much investigated as the opposite one of defect of oxygen, which from the point of view of classification of organisms into anaerobic and aerobic organisms has had a considerable amount of labour bestowed upon it by many investigators, we have carried our previous experiments somewhat further and tested the behaviour of our full stock of laboratory organisms toward increased amounts of oxygen in the atmosphere in which the organisms are grown.

The only other organism in our list specially affected by the increased oxygen percentage was Bacillus pestis, the growth of which we found to be invariably arrested by over 60 per cent. of oxygen in the atmosphere.

The Bacillus pestis is not killed by the oxygen at least after two or three days' exposure-we have not followed it further than this time interval-for, if the tubes which have not grown at all in the oxygen, while their fellows in air have grown quite well, be now removed to air, they commence growing and grow quite well.

That the $B$. pestis should behave somewhat similarly in regard to oxygen habits to the $B$. tuberculosis, is somewhat interesting in view of the occurrence of both plague and tuberculosis in ill-ventilated dwellings. If we add to these two organisms the Staphylococcic group, affected in somewhat lessened degree, we have the short list of what we might perhaps term the oxyphobic organisms from a fairly long list which we have tried to grow under similar and otherwise favourable conditions in air and in increased oxygen percentage respectively.

The complete list of the organisms which we have attempted to test in this way is as follows:-

\footnotetext{
1. Bacillus tuberculosis (human, bovine, and avian).

2. Bacillus coli communis.

3. Bacillus typhosus.

4. Bacillus dysenteriae (Flexner).

5. Bacillus dysenteriae (Kruse).

6. Bacillus dysenteriae (Shiga).

7. Bacillus mallei.

8. Bacillus gaertner.

9. Bacillus prodigiosus.

10. Bacillus liquefaciens fuorescens.

11. Bacillus pneumoniae.

12. Bacillus pyocyaneus.

13. Bacillus of hog cholera.
}

\author{
14. Bacillus diphtheriae. \\ 15. Bacillus anthracis. \\ 16. Bacillus metchnikoff. \\ 17. Bacillus megatherium. \\ 18. Bacillus pestis. \\ 19. Staphylococcus pyogenes aurcus. \\ 20. Staphylococcus pyogenes albus. \\ 21. Staphylococcus pyogenes citreus. \\ 22. Vibrio cholerae. \\ 23. Sarcina lutea. \\ 24. Sarcina aurentiaca. \\ 25. Oidium albicans. \\ 26. Proteus vulgaris.
}


The organisms were sown upon slants of agar plugged in the usual way with cotton wool. Both the tubes intended to be grown in higher amounts of oxygen, and those in ordinary air, were placed in beakers standing in two bell-jars of similar size, and of about 3 litres capacity. Each bell-jar had a tubulure at the top, fitted with a rubber cork pierced by two glass tubes provided with stop-cocks, by means of which the bell-jar could be exhausted and oxygen afterwards allowed in. Each bell-jar had a ground flanged bottom or rim fitting on to a ground glass plate in the ordinary way, and the grinding was most accurate to avoid leakage over the lengthy periods of experimentation. Further, to avoid leakage, and so lowering of the oxygen pressure, a mound of putty was constructed of about one inch wider diameter than the base of the belljar, and mercury was run into this annular space so as to make a mercury seal; but no mercury was in the air-space within the bell-jar. In the case of the organisms to be grown in air, the bell-jar and other fittings were only set up as a dummy of the other experiment so as to make physical conditions of ventilation, air movement, drying, \&c., alike for both sets of tubes, but the atmosphere within was not altered in any way. The amount of oxygen in the other bell-jar to contain the tubes to be grown in oxygen was enriched by pumping out the air by means of a water pump, and then admitting oxygen from a reservoir of that gas. When a high percentage of oxygen was required, the exhaustion and re-filling with oxygen was repeated several times. The oxygen used was manufactured in the laboratory from potassium permanganate. The atmosphere in the bell-jar was analysed, usually for oxygen and carbondioxide, at the beginning and end of the experiment. The percentage of carbon-dioxide was kept down very low by a flat dish of soda-lime included with the tubes under each bell-jar.

\section{Description of EXPERIMENTS}

The numbering of experiments is carried on from the previous paper in which EXPERIMENTS I to $\mathrm{XV}$ are recorded.

Experiment XVI.-The increased oxygen percentage bell-jar contained at the end of the experiment 50.7 per cent. of oxygen; controls in atmospheric air as above described. Two tubes of each organism in oxygen bell-jar and in air bell-jar.

Organisms experimented with were $B$. coli, B. typhosus, B. diphtheriae, B. dysenteriae (Flexner, Kruse, Shiga), S. albus, S. aureus, $S$. citreus. 
Duration of experiment about thirty hours.

Results.-B. coli, B. typhosus, B. diphtheriae showed no differences as between air and oxygen; as also $S$. citreus and $B$. dysenteriae (Flexner); there was possibly slight inhibition of growth in the cases of B. dysenteriae (Shiga) and B. dysenteriae (Kruse). The two Staphylococci $S$. aureus and $S$. albus, however, showed good growths in the airgrown controls, and no growth whatever in the oxygen-grown tubes.

ExPERIMENT XVII.-Oxygen percentage at end 50, the bell-jar having been partially evacuated and charged only once at beginning. Duration of experiment about fifty hours. Organisms used were $S$. aureus and S. albus, and B. dysenteriae (Kruse and Shiga), two tubes of each in air and in increased oxygen.

Results.-All the controls in air had grown well. The Kruse and Shiga in oxygen had also grown well; but the two Staphylococci, aureus and alba, had not grown in the least.

Experiment XVIII.-Bell-jar partially exhausted and re-filled with oxygen for four times; final percentage of oxygen was 84 per cent.

The following organisms were used, two tubes each in air control and in the oxygen, viz.:-B. mallei, B. gaertner, B. prodigiosus, M. agilis, B. pneumoniae, B. pyocyaneus, Hog Cholera, Sarcina lutea, Sarcina aurentiaca, Oidium albicans, Proteus vulgaris.

The controls in this case were grown in the same incubator, but not under a bell-jar.

Duration of the experiment about forty-five hours.

RESULTs.-No differences were. observable in any of these organisms between amount of growth in oxygen and that in air.

Experiment XIX.-Oxygen percentage raised to 77 per cent.; two tubes of each organism in usual controls, and two in oxygen. Organisms tested:-B. anthracis, B. metchnikoff, B. megatherium, and B. diphtheriae. Duration about forty-eight hours.

Results.-Equal growths in all cases in air and in oxygen.

Experiment XX.-The two Staphylococci were here grown in a lower percentage of oxygen (but still higher than air) to attempt to settle lower limit of oxygen inhibition. Richness of oxygen was 45 per cent., duration of experiment about seventy hours. Two tubes each in air control and in the oxygen mixture.

REsults.-Staphylococcus albus: Both controls show good growth; one oxygen tube shows good growth, the other a poor growth. 
Staphylococcus aureus: Both controls show poor growth; one oxygen tube shows poor growth, the other shows hardly any growth at all.

Experiment XXI.-Oxygen percentage 91 ; controls as usual, two tubes in each lot. Duration of experiment about three days. Organisms used: Bacillus pestis and Vibrio finkler (Prior).

Results.-Bacillus pestis: The two controls in air show fair growths; the two oxygen tubes show no growth. V. finkler (Prior): All four tubes show good growth.

ExPERIMENT XXII.-Oxygen percentage 90; controls as usual; two tubes in oxygen and two in air. Bacillus pestis used only; duration two days.

Results.-Both controls show good growth; neither of the two oxygen tubes show any growth at all.

Experiment XXIII.-Oxygen percentage 75 ; controls as usual, three tubes in air and three in oxygen. The organism used was a strain of Streptococcus. Duration of experiment two days.

REsults.-All six tubes show a good growth; there may just be a slight difference in favour of the air-grown tubes.

EXPERIMENT XXIV.-Oxygen percentage 93; controls as usual; two tubes of each organism in oxygen, and two in air. Organisms tested: Bacillus pestis and above strain of Streptococcus. Duration of experiment two days.

RESULTS.-All four tubes of Streptococcus show practically equal growth. The two air-grown tubes of Bacillus pestis show good growth; the two oxygen-grown tubes show no growth.

Experiment $\mathrm{XXV}$.- Oxygen percentage intermediate at about 60 per cent. to test the sensitive organisms for range; two tubes each in oxygen and in air. Organisms tested: Bacillus pestis, Staphylococcus albus and Staphylococcus aureus. Duration of experiment three days.

Results.-Bacillus pestis: The two oxygen tubes show no growth; the two air tubes show a good growth. Staphylococcus albus: The two controls show a good growth; the oxygen tubes (60 per cent.) show a fair growth, roughly about half as great as the air tubes. Staphylococcus aureus: A similar result to the albus.

In order to test whether the plague organism was killed by the increased oxygen, or only inhibited in its growth, the two tubes previously in oxygen, and showing no growth, were now placed in the 
incubator for two further days in air, when a good growth appeared, showing that the organism had only been inhibited and not killed by the previous sojourn in oxygen ( 60 per cent.).

Experiment XXVI.-Oxygen percentage at 78.4. Tubes of Bacillus pestis and of Staphylococcus albus, two each in air and in oxygen. Kept for two days.

Results.-Bacillus pestis: Controls show good growth; oxygen tubes show not a trace of growth. Staphylococcus albus: Controls show excellent growth, and oxygen tubes just a trace of growth.

All four of the oxygen tubes were now taken and placed in the ordinary way in air in the incubator. At the end of two days all four had grown normally equally with previous growth of the controls. Hence, the growth had only been kept in abeyance by the oxygen.

Experiment XXVII.-Oxygen percentage 88; two tubes each of B. pestis, S. albus and S. aureus, in air and in oxygen respectively. Duration three days.

Results.-B. pestis: Controls in air show good growth; oxygen tubes no growth. S. albus: The oxygen tubes do show a distinct growth, but not to be compared with air-grown controls. S. aureus: The oxygen tubes show a growth nearly as good as control. Accordingly, this experiment, like the next, gives an exceptional result with $S$. aureus in oxygen. Bacillus pestis, however, uniformly refuses to grow in oxygen throughout, although tried on seven occasions with two tubes on each occasion.

As before, the negative tubes grew when subsequently tried in air.

Experiment XXVIII.-Oxygen percentage 86. Two tubes of Bacillus pestis, Staphylococcus aureus, and Staphylococcus albus, respectively, in oxygen, and two tubes of each organism in air. Duration of experiment two days.

REsults.-All controls show good growth. The plague organism, as usual, has not grown at all in the oxygen; the Staphylococci, both albus and aureus, do show a fair growth, even in the oxygen, but not so luxuriant as in the air.

\section{Conclusions}

1. A list of twenty-six organisms has been tested as to comparative growth in atmospheres of air, and of air enriched in high degree by oxygen. 
2. Two organisms stand out prominently as oxyphobic, namely, those of tuberculosis and of plague, and the only others affected adversely by the increased oxygen are the organisms of the Staphylococcic group, particularly Staphylococcus pyogenes aureus and Staphylococcus pyogenes albus.

3. The Bacillus tuberculosis is not only arrested in growth, but is killed by the three weeks exposure to the higher percentage of oxygen necessary to determine the question of comparative growth. The Bacillus pestis, although it uniformly refused to grow on seven occasions with percentages of oxygen of $91,90,93,60,78,88$, and 86 , respectively, was not killed by these amounts during the two to three days demanded for the decision of the experiment, since the tubes grew afterwards when brought into air cultivation. It has not been determined whether longer exposure for two or three weeks would kill the organism. 